\title{
Criminologie
}

\section{Délinquance et immigration en France : un regard sociologique}

\section{Laurent Mucchielli}

Volume 36, numéro 2, automne 2003

Le construit de l'ethnicité en criminologie

URI : https://id.erudit.org/iderudit/007865ar

DOI : https://doi.org/10.7202/007865ar

Aller au sommaire du numéro

\section{Éditeur(s)}

Les Presses de l'Université de Montréal

\section{ISSN}

0316-0041 (imprimé)

1492-1367 (numérique)

Découvrir la revue

\section{Citer cet article}

Mucchielli, L. (2003). Délinquance et immigration en France : un regard sociologique. Criminologie, 36(2), 27-55. https://doi.org/10.7202/007865ar

\section{Résumé de l'article}

En France, comme dans la plupart des pays occidentaux, la figure de l'immigré est fortement associée à celle du délinquant, dans les représentations collectives et dans les discours médiatico-politiques sur l'« insécurité ». Cette association se scinde en deux problématiques : la délinquance des étrangers et celle des « jeunes issus de l'immigration ». Cet article se propose de faire une synthèse critique des connaissances sur ces deux questions, à partir des données administratives et des travaux sociologiques de nature quantitative et qualitative. L'examen rigoureux des statistiques policières ne permet pas de mesurer la délinquance des étrangers. Il invite toutefois à distinguer une délinquance professionnelle des étrangers non résidents d'une délinquance d'étrangers résidents qui s'apparente aux vols et aux violences physiques classiquement observés dans les couches les plus pauvres de la population. Les travaux sociologiques permettent ensuite de mettre en évidence le fait qu'une sur-représentation des jeunes issus de l'immigration africaine dans la population délinquante juvénile peut être observée localement mais non de façon uniforme sur le territoire national. Ce constat amène alors à rechercher les effets de contextes locaux qui favorisent le développement de cette spécificité, dans une perspective tant sociologique que psychosociologique. 


\title{
Délinquance et immigration en France : un regard sociologique ${ }^{1}$
}

\author{
Laurent Mucchielli \\ CNRS - CESDIP \\ France \\ mucchiel@ext.jussieu.fr
}

\begin{abstract}
RÉSUMÉ • En France, comme dans la plupart des pays occidentaux, la figure de l'immigré est fortement associée à celle du délinquant, dans les représentations collectives et dans les discours médiatico-politiques sur l'«insécurité». Cette association se scinde en deux problématiques : la délinquance des étrangers et celle des «jeunes issus de l'immigration ». Cet article se propose de faire une synthèse critique des connaissances sur ces deux questions, à partir des données administratives et des travaux sociologiques de nature quantitative et qualitative. L'examen rigoureux des statistiques policières ne permet pas de mesurer la délinquance des étrangers. Il invite toutefois à distinguer une délinquance professionnelle des étrangers non résidents d'une délinquance d'étrangers résidents qui s'apparente aux vols et aux violences physiques classiquement observés dans les couches les plus pauvres de la population. Les travaux sociologiques permettent ensuite de mettre en évidence le fait qu'une sur-représentation des jeunes issus de l'immigration africaine dans la population délinquante juvénile peut être observée localement mais non de façon uniforme sur le territoire national. Ce constat amène alors à rechercher les effets de contextes locaux qui favorisent le développement de cette spécificité, dans une perspective tant sociologique que psychosociologique.
\end{abstract}

ABSTRACT • In France as in most western countries, the immigrant is strongly associated with delinquency in collective representations and in media and political discourses concerning «insecurity». This association can be decomposed into two distinct concerns : the delinquency of foreigners and the delinquency of French youth born of immigrants. This article proposes a critical survey of French research on these questions, based on administrative data and sociological research, both qualitative and quantitative. The rigorous examination of police statistics does not allow for the measurement of foreigners' delinquency. It does, however, invite us to distinguish between the professional delinquency of non resident foreigners and the delinquency of resident foreigners, such as theft

1. L'auteur tient à remercier Michel Kokoreff, René Lévy, Philippe Robert et Renée Zauberman pour leurs commentaires sur des versions antérieures de ce texte. 
and physical violence, a type of delinquency classically observed in the poorest part of the population. Sociological research allows us, then, to emphasize the fact that an overrepresentation of youth born of African immigrants among juvenile delinquents can be observed in some places but not as a general and uniform process. This statement of fact leads us to try to discover the local processes that favour the development of this local variation, from both sociological and psycho-sociological points of view.

En France, tel qu'il se développe depuis maintenant plus de vingt-cinq ans, le débat public sur «l'insécurité» tourne autour de quelques thèmes centraux dont l'un des principaux est la relation supposée entre délinquance et immigration. Cette relation est elle-même double. Elle vise, d'une part, les étrangers proprement dits et, en leur sein, plus particulièrement ceux qui séjournent clandestinement sur le territoire national et, d'autre part, les «jeunes issus de l'immigration», pour la plupart de nationalité française, et en leur sein, plus particulièrement les jeunes d'origine maghrébine et noire africaine. Longtemps, cette relation a été traitée sur un mode essentiellement idéologique et moralisateur. Devant l'accusation portée sur le plan politique par la droite et l'extrême droite, répondaient la gauche et les mouvements anti-racistes. Le débat était nettement clivé. Toutefois, au cours des années 1990, ce clivage s'est en partie estompé (Mucchielli, 2002 : 30-32). Au tournant des années 1980 et 1990, «l'affaire du foulard islamique», l'apparition des «émeutes urbaines» puis le contexte de la (première) guerre du Golfe ont beaucoup accrû la peur du monde arabe et de l'Islam en France (Cesari, 1997) 2 . Ensuite, le tournant politique du Parti socialiste et du gouvernement Jospin (1997-2002), sur la question de la «sécurité» et en particulier de la délinquance juvénile, a tout à la fois atténué fortement la critique traditionnelle de gauche sur ces questions et permis l'expression beaucoup plus libre de discours désignant comme principaux responsables les étrangers et les jeunes issus de l'immigration. Symbole de cette petite révolution, des figures de la lutte antiraciste telles que le président de SOS Racisme (Le Figaro, 25 avril 2002) et le père Delorme (Le Monde,

2. Ce que l'on appelle «l'affaire du voile islamique» s'est déroulée à la fin de l'année 1989. L'histoire a débuté avec la décision d'un proviseur d'un collège de la région parisienne d'exclure de son école pour "atteinte à la laïcité et à la neutralité de l'école publique» trois jeunes filles qui refusaient d'enlever leur voile en classe. La médiatisation de cet événement l'a progressivement transformé en événement politique national, de nombreux intellectuels prenant également part à un débat qui s'étendra de la laïcité de l'école à la place de la religion islamique en France et, plus généralement encore, à la question dite de «l'intégration des immigrés» (Gaspard et Khosrokhavar, 1995). 
3 décembre 2001), acteur important des actions collectives menées au début des années 1980 par les jeunes d'origine maghrébine, ont ellesmêmes réclamé publiquement que l'on «brise le tabou» en reconnaissant l'existence d'une «sur-délinquance» des jeunes issus de l'immigration.

Cette évolution serait heureuse si elle ouvrait sur un débat intellectuel, si elle permettait à l'argumentation et à la démonstration contextualisante de prendre le pas sur l'émotion et le sens commun. Ce n'est guère le cas. Après un bref rappel des discours dominants dans le débat public et des formes de dénonciation du lien entre délinquance et immigration, nous tenterons cependant de faire le point des connaissances sur cette question et d'expliciter ce que peut être le raisonnement sociologique en cette matière.

\section{Une nouvelle imputation : la « dérive mafieuse des cités »}

Le tableau des nouvelles formes de délinquance que dressent aujourd'hui nombre d'intervenants dans le débat public est en substance le suivant : nous serions face à une jeunesse désocialisée, déscolarisée, sans repères moraux et sociaux, pourvue de parents «démissionnaires». Pire : ces jeunes seraient massivement toxicomanes et, pour consommer comme pour s'enrichir, ils deviendraient fatalement de jeunes trafiquants de drogues qui ne tarderaient pas à s'organiser en bandes délinquantes et armées, organisant toute une économie souterraine dans leurs cités et terrorisant les territoires environnants. C'est ainsi que les incendies de voitures serviraient uniquement à faire disparaitre les voitures volées, tandis que les émeutes ne seraient en rien l'expression d'un sentiment d'injustice, mais simplement un moyen de tenir à distance la police pour mieux protéger les trafics. Et dans cette véritable organisation délinquante, les plus âgés initieraient les plus jeunes. Ainsi se mettrait progressivement en place un véritable «système mafieux» organisé autour de quelques «familles délinquantes» contrôlant peu à peu tout un quartier et utilisant les jeunes pour se protéger de la police ${ }^{3}$. Ensuite, il ne s'agirait pas de n'importe quelles familles, mais des maghrébines. Il y aurait à cela des raisons «culturelles» :

3. C'est la démonstration proposée notamment par la commissaire Bui-Trong (1998) des Renseignements généraux, le commissaire divisionnaire Bousquet (1998) du Syndicat des commissaires et des hauts fonctionnaires de la police nationale, et le tandem formé par un conseil privé en sécurité et un journaliste enseignant à l'Institut de criminologie de l'Université de Paris 2-Assas (Bauer et Raufer, 1998). 
«jugé peu dangereux par la tradition et la culture de populations d'origine maghrébine, légitimé par son impact économique positif, le trafic de haschisch structure les emplois du temps et soutient la capacité de consommation du quartier. Facteur de paix sociale, il maintient sur le quartier le voile du silence mafieux» (Bousquet, 1998 : 119). Cette évocation d'une consommation (et par la suite d'un trafic) de drogues qui serait en quelque sorte légitimée par «la culture de populations d'origine maghrébine» constitue pourtant une erreur. En réalité, un seul pays du Maghreb est producteur de haschisch : le Maroc (Observatoire géopolitique des drogues, 1996 : 113-115). Le problème n'est donc pas maghrébin en général, mais marocain en particulier. En outre, il n'est pas lié à la «culture» du Maroc, mais à son histoire coloniale et au fonctionnement actuel de son économie 4 .

Autre exemple : lorsqu'un de ces intervenants (Raufer) est entendu sur la délinquance par la Commission sénatoriale sur la décentralisation, le 28 mars 2000, le rapport public enregistre la déclaration de cet auteur selon lequel «le tabou majeur en matière de délinquance urbaine concernait l'origine des auteurs d'infractions. Il a fait part d'une enquête des renseignements généraux mettant en évidence que sur 3000 auteurs de violences urbaines, une cinquantaine seulement avaient un patronyme "gaulois"». Au même moment, sur le site Internet de Claude Goasgen, député parisien du parti Démocratie libérale, à côté de pages consacrées notamment à la dénonciation de la «réalité cachée » de l'immigration, le même auteur s'exprime sur le lien entre délinquance et immigration : «ces liens sont parfaitement mécaniques : une population jeune, masculine et déracinée est par essence plus criminogène que de vieilles dames vivant au village, un enfant de quatre ans comprend cela». Le problème est ici que, dans leur immense majorité, les jeunes en question sont nés en France et ne connaissent que la France. Ils ne sont donc nulle-

4. Ce n'est qu'à la fin du XIX ${ }^{\mathrm{e}}$ siècle que la culture du cannabis a pris une véritable ampleur au Maroc, surtout à l'époque des protectorats français et espagnol. L'une des conséquences du protectorat français fut en effet la création d'une «Régie des tabacs et du kif», société au capital majoritairement français, qui organisa la production de masse du haschisch. En 1925, la France ratifia toutefois la convention de Genève interdisant la culture de ce qui était désigné comme une drogue, mais pas l'Espagne. De plus, dans la zone française, l'interdiction ne fut pas réellement appliquée (on se contenta souvent de donner des amendes aux contrevenants). À partir de 1956 (date de l'indépendance et de la réunification du Royaume), les autorités marocaines auront ainsi les pires difficultés à lutter contre une économie théoriquement illégale, qui constitue pourtant une ressource financière essentielle pour près de 5 millions de paysans de ce pays pauvre. En fait de problème «culturel», nous sommes devant un problème économique et, pour les dirigeants marocains, un problème politique. 
ment «déracinés» comme ont pu l'être parfois leurs parents, en particulier lorsqu'ils provenaient d'un milieu rural ${ }^{5}$. Mais cette erreur est sans doute logique dans un système de pensée qui, derrière ce thème ancien du «déracinement», attribue à l' "étranger» une altérité irréductible et un potentiel délinquant intrinsèque («ontologique», disait Sayad (1997)) $)^{6}$.

Devant ces imputations, et sans présumer du bien-fondé éventuel de tel ou tel élément de leur argumentation générale, tournons-nous à présent vers les éléments de connaissance à disposition, en commençant par la question de la délinquance des étrangers.

\section{Une surdélinquance des étrangers?}

\section{Les informations limitées contenues dans les données policières}

La source statistique la plus connue en matière de délinquance est celle constituée par les forces de police et de gendarmerie. De fait, c'est la moins incomplète des données administratives que l'on puisse utiliser ${ }^{7}$.

5. Sur les conséquences psychologiques et sociales de ce déracinement chez les premiers arrivants, voir notamment Sayad (1981) et les travaux de psychologie interculturelle développés par Carmel Camilleri et son équipe depuis plus d'une vingtaine d'années.

6 . Le thème du «déracinement» constitue une explication relativement courante de la délinquance des étrangers, que l'on retrouve par exemple jusque dans le recueil de statistiques du ministère de l'Intérieur qui, en 1975, concluait ainsi sa description de la surreprésentation apparente des étrangers dans la délinquance enregistrée : «Ces conclusions n’autorisent aucun jugement discriminatoire à l'encontre des immigrés. Comment des hommes déracinés, transportés dans un autre mode de vie et soumis à l'exemple de la contagion d'une criminalité plus importante que celle de leur propre pays ne succomberaient-ils pas en plus grand nombre que les nationaux? C'est le contraire qui serait étonnant» (Ministère de l'Intérieur, 1976 : 86). Historiquement, ce thème est issu de la tradition de pensée nationaliste et trouve ses origines modernes à la fin du XIX ${ }^{\mathrm{e}}$ siècle, notamment dans l'œuvre de Maurice Barrès, auteur du roman fameux Les déracinés en 1897 (Sternhell, 1985). Son influence à l'époque débordait cependant le cadre de la pensée nationaliste (et raciste) proprement dite pour s'étendre à des courants plus simplement traditionalistes, faisant de l'attachement à la terre, au village et à la famille élargie, une condition de la bonne marche d'une société. L'influence de ce courant de pensée se retrouve aussi dans les sciences sociales de l'époque, notamment chez les disciples de Frédéric Le Play (Veitl, 1994). Il n'a d'ailleurs pas disparu des sciences sociales au long du $\mathrm{XX}^{\mathrm{e}}$ siècle, ainsi que l'a souligné Noiriel (1988 : chapitre 1).

7. On ne commettra pas l'erreur fréquente (par exemple chez Bauer et Raufer (1998: 5 et 10)) consistant à tirer des conclusions de l'analyse de la population carcérale. Les étrangers sont surreprésentés en prison pour au moins quatre raisons : 1) il s'agit souvent de personnes dont le seul délit est le défaut de papiers en règle, infractions qui, par définition, ne sont commises que par les étrangers (ce sont les clandestins); 2) ils sont surreprésentés parmi les auteurs poursuivis par la police pour certaines infractions actuellement sévèrement condamnées (en particulier le trafic de drogue); 3) ils sont surreprésentés parmi les populations les plus pauvres qui sont elles-mêmes surreprésentées dans l'ensemble des auteurs d'atteintes aux biens et aux 
Cette statistique livre cependant peu d'informations intéressant le sociologue. En l'occurrence, elle donne trois informations démographiques sur les personnes mises en cause (c'est-à-dire les personnes identifiées par la police comme auteurs d'infractions et faisant l'objet de procèsverbaux transmis aux parquets) : leur sexe, leur statut (majeur ou mineur) et leur nationalité (française ou étrangère). On n'y cherchera donc pas d'information sur la délinquance des personnes issues de l'immigration mais simplement sur la délinquance des personnes de nationalité étrangère. Dans ce cadre, l'intérêt comme la limite de cette source policière sont bien connus (Tournier et Robert, 1990; Robert et al., 1994). L'intérêt est d'offrir année après année une vue d'ensemble sur les résultats de l'activité des services de police et de gendarmerie. La limite est contenue dans l'énoncé même : il s'agit des résultats de l'activité des policiers et des gendarmes, non de celle des délinquants. Les deux ne sont évidemment pas sans rapport et la première enregistre bien une partie de la seconde, mais une partie seulement. Pour simplifier, la statistique de police opère un tri dans la réalité délinquante et, pour juger de la pertinence des données de cette statistique, il faut donc comprendre les logiques de ce tri ${ }^{8}$.

La statistique de police et de gendarmerie est alimentée de deux façons très différentes : par ce que les victimes viennent signaler à la police et par ce que les policiers trouvent eux-mêmes (qu'il s'agisse d'interventions de police urbaine en flagrant délit ou bien, à l'opposé, des résultats d'enquêtes de moyen ou long terme menées par la police judiciaire). Rien dans les enquêtes menées auprès des victimes ne permet aujourd'hui de savoir si la nationalité (voire même la couleur de peau) des délinquants influe sur le choix des victimes de signaler ou non à la police les infractions qu'elles-mêmes ou leurs proches ont subies. Mais il n'en va pas de même dans l'attitude des policiers. La question du «contrôle au faciès» est classique. Qu'est-ce à dire? Que les policiers, en tant que personnes, sont racistes? Le constat est très fréquemment dressé par les personnes contrôlées lorsqu'elles portent les signes physiques d'une origine africaine

personnes; 4) les recherches ont établi depuis longtemps qu'à infraction égale, les étrangers sont condamnés plus sévèrement que les nationaux (Robert, 1995; Tournier, 1997; Mary et Tournier, 1998).

8. Pour mémoire, on rappellera tout de même que la plupart des points discutés ici avaient été au moins pressentis dès les premiers travaux sociologiques portant sur la construction des statistiques administratives. Deux articles de Thorsten Sellin $(1928 ; 1935)$, notamment, méritent de ce point de vue relecture. Pour une discussion des travaux américains de l'entre-deuxguerres sur le lien entre délinquance et immigration et sur les théories du «conflit de culture», voir Brion et Tulkens (1998) et Robert (1999). 
et en particulier maghrébine, qu'elles soient ou non auteurs d'un acte de délinquance. Le problème est cependant beaucoup plus compliqué et, en un sens, beaucoup plus prégnant. Deux spécialistes de la police le résument si justement que nous préférons les laisser parler plutôt que les paraphraser :

Tous les chercheurs qui ont observé de près les pratiques policières, en France comme à l'étranger, concluent à la réalité d'un discours raciste généralisé, qui constitue pour les policiers une véritable norme à laquelle il est difficile, lorsqu'on est policier de base, d'échapper et plus encore de s'opposer. Le caractère normatif de ce racisme policier en fait avant tout un élément de la culture policière, distinct du racisme ambiant ou de celui des couches sociales dont les policiers sont issus, et qui n'a pas un caractère de construction idéologique ou doctrinaire. [...] le racisme policier a essentiellement un caractère réactif. On n'entre pas dans la police parce qu'on est raciste, on le devient à travers le processus de socialisation professionnelle. En d'autres termes, l'habitude de juger les individus en fonction de leurs caractéristiques ethniques supposées s'acquiert sur le tas, au cours de la socialisation professionnelle. [...] les représentations racistes ont un caractère opératoire, en ce qu'elles permettent de différencier des individus. Dans la pratique, en orientant la vigilance policière, elles participent du mécanisme de la prédiction créatrice. Elles constituent en quelque sorte des instruments de travail et font partie de cet ensemble de connaissances pratiques qui forment l'arrière-plan, la référence du travail policier. Le recours aux attributs ethniques a pour les policiers un caractère fonctionnel, au même titre que l'âge ou le sexe, dans la mesure où la police de la rue renvoie avant tout à une conception de la normalité conçue comme adéquation d'un type de population, d'un espace et d'un moment donnés. Tout décalage entre ces trois paramètres déclenche le soupçon policier et peut déboucher sur une intervention (Lévy et Zauberman, 1998 : 293-294; voir aussi Monjardet, 1996).

La chose vaut pour les types africains, comme elle vaut dans un autre contexte pour les Gitans (Zauberman, 1998). Dès lors, le problème serait d'évaluer l'impact de ces pratiques professionnelles des policiers. En l'absence d'études locales systématiques - dont on devine les difficultés de réalisation concrète - cette évaluation est impossible à faire avec précision. Toutefois, on dispose de nombreux éléments pour étayer le mécanisme du tri sélectif des policiers.

Tandis qu'environ quatre millions d'infractions sont recensées par la police et la gendarmerie chaque année (dans la période des années 1990), seul un quart d'entre elles sont élucidées et donnent lieu à des mises en cause de personnes. Les données policières françaises ont l'intérêt de publier le détail de ce taux d'élucidation des affaires (tableau 1), ce qui 
permet de constater l'écart entre la masse des faits constatés et celle des faits élucidés : «en passant de l'une à l'autre, un contentieux fait peau de chagrin, celui des vols et des destructions, autrement dit la plus grande partie des plaintes des particuliers. Par contrecoup, les contentieux dont la découverte dépend surtout de l'initiative policière occupent une place relativement disproportionnée parmi les mis en cause» (Robert et al., 1994 : 60). Appliqués à notre objet, ces constats sont essentiels pour deux raisons.

D'une part, si la population des personnes mises en cause est surtout alimentée par les initiatives policières, sachant que, en matière de petite

TABLEAU 1

Comparaison des faits constatés et des faits élucidés par la police en $\mathbf{2 0 0 0}$

\begin{tabular}{|l|r|c|}
\hline Nature de l'infraction & $\begin{array}{c}\text { Nombre de } \\
\text { faits constatés }\end{array}$ & $\begin{array}{c}\text { \% de faits } \\
\text { élucidés }\end{array}$ \\
\hline Total des vols & 2334696 & 11,25 \\
dont vols à l'étalage & 57379 & 86,56 \\
dont vols à la tire* & 100774 & 3,03 \\
dont vols de voitures & 301539 & 7,2 \\
dont vols à la roulotte** & 670177 & 5,22 \\
dont vols avec violence sans arme à feu sur voie publique & 96080 & 14,04 \\
\hline Cambriolages & 370993 & 9,2 \\
\hline Escroqueries, faux et contrefaçons & 317044 & 53,97 \\
\hline Menaces et chantages & 48632 & 62,91 \\
\hline Coups et blessures non mortels & 106312 & 71,8 \\
\hline Viols & 8458 & 75,46 \\
\hline Homicides et tentatives & 2166 & 78 \\
\hline Proxénétisme & 368 & 94,29 \\
\hline Trafic de drogues & 4254 & 117,3 \\
\hline Usage/revente de drogues & 14058 & 107,83 \\
\hline Usage de drogues & 78305 & 102,87 \\
\hline Violences et outrages à dépositaires de l'autorité & 39265 & 96,8 \\
\hline Ports et détention d'armes prohibées & 21984 & 100,4 \\
\hline
\end{tabular}

Source : ministère de l'Intérieur.

Note de lecture : le taux d'élucidation est parfois supérieur à $100 \%$ pour des raisons qui peuvent tenir à des reports d'une année sur l'autre et/ou à des doubles comptages dans différents services de police. Cela concerne essentiellement les affaires de stupéfiants.

* Pick-pockets.

** Vols avec effraction dans un véhicule moteur. 
délinquance, les policiers privilégient le plus souvent le flagrant délit sur l'enquête (Lévy, 1987), les mécanismes de la discrimination policière peuvent donc jouer massivement et les étrangers seront par définition surreprésentés de façon artificielle dans la population des mis en cause. Ceci est sans doute particulièrement vrai dans le cas des vols et des agressions commis sur la voie publique.

D'autre part, si le taux d'élucidation varie beaucoup selon la nature de l'infraction, il faut se demander si les étrangers ne sont pas surreprésentés pour certaines infractions mieux élucidées que d'autres. Et c'est précisément le cas de la plupart d'entre elles (à l'exception du vol à la tire qui n'est quasiment pas élucidé par la police mais, on y reviendra, par les services de sécurité des grands magasins). Dans l'ordre croissant d'importance numérique, citons le proxénétisme (occasionnant la mise en cause de 225 étrangers, élucidé dans environ $94 \%$ des cas), les homicides (occasionnant la mise en cause de 291 étrangers, élucidés dans environ $78 \%$ des cas), les viols (occasionnant la mise en cause de 703 étrangers, élucidés dans environ $75 \%$ des cas), le trafic de drogues (occasionnant la mise en cause de 1992 étrangers, élucidé dans environ $117 \%$ des cas), les ports et détentions d'armes prohibées (occasionnant la mise en cause de 2025 étrangers, élucidés dans environ $100 \%$ des cas), les violences et outrages à dépositaires de l'autorité (occasionnant la mise en cause de 3724 étrangers, élucidés dans environ $97 \%$ des cas), les vols à l'étalage (occasionnant la mise en cause de 10737 étrangers, élucidés dans environ $87 \%$ des cas) et enfin les coups et blessures non mortels (occasionnant la mise en cause de 11747 étrangers, élucidés dans environ 72\% des cas).

Ces analyses statistiques globales peuvent être complétées par des études plus précises, qu'elles soient de nature qualitative ou quantitative. Par exemple, une étude réalisée sur les trafics de drogues à la frontière francoespagnole souligne à quel point l'implication au plus haut niveau de jeunes hommes blancs issus de milieux aisés est inconnue de la police qui se focalise sur les revendeurs issus pour la plupart de la communauté gitane (Missaoui et Tarrius, 1999). Plus globalement, les enquêtes réalisées auprès des collégiens et des lycéens soulignent que ces derniers consomment du cannabis dans tous les milieux sociaux, de façon parfois même plus fréquente dans les milieux aisés (Choquet et Ledoux, 1994). Or, les personnes mises en cause par la police pour simple usage de cannabis sont massivement des jeunes issus de milieux populaires, sans doute très souvent d'origine africaine (Barré et Godefroy, 1999). Les conséquences du «tri ethnique» et du tri social opérés par les policiers sont donc assez claires. 
En plus de tout cela, on peut enfin observer un tri qui s'observe dans les pratiques des agents des sociétés privées de surveillance. Dans leur recherche sur les pratiques de sécurité dans les grandes surfaces, Ocqueteau et Pottier (1995 : 138) constatent ainsi que, lorsqu'un voleur à l'étalage est surpris, il a beaucoup plus de chances d'être signalé à la police (par opposition au règlement à l'amiable) lorsqu'il est étranger.

Pour toutes ces raisons, les données policières doivent être analysées avec la plus grande prudence. Examinons donc à présent en détail la seule information statistique fournie par cette source : la part des étrangers dans l'ensemble des personnes mises en cause par la police et la gendarmerie (tableau 2), avant de tenter de l'interpréter.

\section{Le poids global et les grandes caractéristiques des étrangers mis en cause par la police et la gendarmerie}

Calculer le poids global des étrangers dans la délinquance et la criminalité élucidées suppose de nouveaux calculs. Au recensement de 1999, la France comptait 60,2 millions d'habitants (58,5 en métropole), dont 3,3 millions d'étrangers et 2,4 millions de Français par acquisition. Pour le seul territoire de la métropole, cela donne cette répartition : 90,4\% de Français de naissance, $4 \%$ de Français par acquisition et 5,6\% d'étrangers (dont 43,5\% de personnes de nationalités africaines et, plus précisément, $35 \%$ de ressortissants de pays du Maghreb) (Lebon, 2000). Une proportion de 5,6\% d'étrangers, voilà donc notre base de comparaison. Mais comparaison avec quoi? Certes pas avec l'ensemble des crimes et délits répertoriés dans les statistiques de police. Il faut en effet écarter des calculs ce que nous appellerons les «infractions administratives» dans lesquelles les étrangers sont par définition fortement surreprésentés. Il s'agit pour l'essentiel : 1) des délits à la police des étrangers, 2) des délits de faux documents d'identité et autres documents administratifs et 3) du délit de travail clandestin (voir aussi la remarque de Desdevises, 1998 : 270-273). Ces infractions écartées, le nombre de personnes étrangères mises en cause par la police et la gendarmerie en l'an 2000 a été d'environ 89 000, ce qui représente un peu moins de $11 \%$ du total des personnes mises en cause cette année-là. Ainsi que l'indique le tableau 3, cette proportion est en réalité remarquablement stable depuis plus d'un quart de siècle, entre 12 et $13 \%{ }^{9}$. Elle constitue donc

9. Avec toutefois une baisse inhabituelle en 2000 due en réalité à un effet de structure (la brusque augmentation de la délinquance enregistrée, dans des catégories d'infractions qui ne concernent pas très significativement les étrangers). La proportion était de 13,1\% en 1998 et 
TABLEAU 2

Part des étrangers dans les mises en cause par la police en 2000*

\begin{tabular}{|c|c|c|c|}
\hline Nature de l'infraction & $\begin{array}{c}\text { Nombre de mis } \\
\text { en cause } \\
\text { étrangers dans } \\
\text { les principales } \\
\text { catégories } \\
\text { d'infraction }\end{array}$ & $\begin{array}{l}\text { Part de chaque } \\
\text { catégorie } \\
\text { d'infraction } \\
\text { dans le total } \\
\text { des étrangers } \\
\text { mis en cause }\end{array}$ & $\begin{array}{l}\text { \% d'étrangers } \\
\text { dans le total des } \\
\text { mis en cause } \\
\text { pour chaque } \\
\text { catégorie } \\
\text { d'infraction }\end{array}$ \\
\hline Total des vols & 34356 & 38,5 & 13,5 \\
\hline dont vols à l'étalage & 10737 & 12 & 20,5 \\
\hline dont vols simples sur particuliers & 4807 & 5,4 & 12,9 \\
\hline dont vols à la roulotte & 2523 & 2,8 & 9,2 \\
\hline $\begin{array}{l}\text { dont vols avec violence sans arme à } \\
\text { feu sur voie publique }\end{array}$ & 2556 & 2,9 & 14,2 \\
\hline dont vols à la tire & 1620 & 1,8 & 44,8 \\
\hline dont vols de voitures & 1484 & 1,7 & 7,6 \\
\hline Cambriolages & 3039 & 3,4 & 9 \\
\hline Escroqueries, faux et contrefaçons & 5980 & 6,7 & 11,7 \\
\hline Coups et blessures non mortels & 11747 & 13,2 & 14,7 \\
\hline Viols & 703 & 0,8 & 12,9 \\
\hline Homicides et tentatives & 291 & 0,3 & 15,9 \\
\hline Proxénétisme & 225 & 0,2 & 40,8 \\
\hline $\begin{array}{l}\text { Infractions contre la famille et } \\
\text { l'enfant }\end{array}$ & 2536 & 2,8 & 8,7 \\
\hline Menaces ou chantages & 3339 & 3,7 & 13,1 \\
\hline Trafic de drogues & 1992 & 2,2 & 27,1 \\
\hline Usage/revente de drogues & 1092 & 1,2 & 7,9 \\
\hline Usage de drogues & 4922 & 5,5 & 6,4 \\
\hline $\begin{array}{l}\text { Incendies, destructions, dégradations } \\
\text { de biens publics ou privés }\end{array}$ & 6290 & 1,1 & 8,6 \\
\hline $\begin{array}{l}\text { Violences et outrages à dépositaires } \\
\text { de l'autorité }\end{array}$ & 3724 & 4,2 & 12,1 \\
\hline Port et détention d'armes prohibées & 2025 & 2,3 & 12,2 \\
\hline $\begin{array}{l}\text { Atteintes aux intérêts fondamentaux } \\
\text { de la nation }\end{array}$ & 35 & 0,04 & 2,9 \\
\hline Autres infractions diverses & 6798 & - & - \\
\hline Total & 89194 & 100 & 10,9 \\
\hline
\end{tabular}

Source : ministère de l'Intérieur.

* Les chiffres et les calculs que nous indiquons excluent les infractions que nous qualifions $d^{\prime}$ « administratives» (voir les explications dans le texte). 
TABLEAU 3

Évolution du pourcentage d'étrangers dans le total des mis en cause pour chaque catégorie d'infraction entre 1975 et 2000

\begin{tabular}{|c|c|c|c|c|c|c|}
\hline Nature de l'infraction & 1975 & 1980 & 1985 & 1990 & 1995 & 2000 \\
\hline Total des vols & 16,3 & 17,7 & 16,9 & 14,8 & 15,2 & 13,5 \\
\hline dont vols à l'étalage & 16,8 & 20,1 & 21,4 & 20,8 & 24,6 & 20,5 \\
\hline dont vols à la roulotte & 21,3 & 18,5 & 17 & 14,4 & 12,3 & 9,2 \\
\hline dont vols à la tire & - & - & - & 43,9 & 43,9 & 44,8 \\
\hline dont vols de voitures & 15,4 & 13,8 & 13,1 & 10,7 & 10,3 & 7,6 \\
\hline Cambriolages & 15,6 & 17,4 & 15 & 10,1 & 11,3 & 9 \\
\hline Escroqueries, faux et contrefaçons & 9,4 & 10,9 & 16,4 & 11,5 & 12,2 & 11,7 \\
\hline Coups et blessures non mortels & - & - & - & 16,6 & 16,2 & 14,7 \\
\hline Viols & 27,2 & 28,8 & 23,4 & 17,2 & 13,3 & 12,9 \\
\hline Homicides et tentatives & 23,3 & 20,3 & 26,1 & 14,8 & 16,6 & 15,9 \\
\hline Proxénétisme & 21,4 & 27,6 & 22,2 & 22 & 22 & 40,8 \\
\hline Infractions contre la famille et l'enfant & 6,5 & 7,1 & 7,1 & 7,4 & 6,9 & 8,7 \\
\hline Menaces ou chantages & - & - & - & 14,2 & 14,1 & 13,1 \\
\hline Trafic de drogues & 69,2 & 74,6 & 61,5 & 38,1 & 30,6 & 27,1 \\
\hline Usage/revente de drogues & - & - & - & 20,1 & 18,1 & 7,9 \\
\hline Usage de drogues & - & - & - & 17,4 & 11,3 & 6,4 \\
\hline $\begin{array}{l}\text { Incendies, destructions, dégradations } \\
\text { de biens publics ou privés }\end{array}$ & 9,9 & 10,3 & 11,4 & 12,3 & 11,4 & 8,6 \\
\hline $\begin{array}{l}\text { Violences et outrages à dépositaires } \\
\text { de l'autorité }\end{array}$ & 13 & 13,4 & 14,1 & 12,7 & 13,7 & 12,1 \\
\hline Port et détention d'armes prohibées & 26,4 & 19,9 & 18,3 & 16,8 & 15,6 & 12,2 \\
\hline $\begin{array}{l}\text { Atteintes aux intérêts fondamentaux } \\
\text { de la nation }\end{array}$ & - & - & - & 2,3 & 1,8 & 2,9 \\
\hline Total & 12,4 & 13,1 & 12,7 & 12,8 & 13,6 & 10,9 \\
\hline
\end{tabular}

Source : ministère de l'Intérieur ; calculs CESDIP.

Note de lecture : le tableau présente quelques disparités dans la mesure où des changements sont intervenus entre 1985 et 1990 dans la présentation des données par le ministère de l'Intérieur. La part des étrangers dans les cambriolages est ainsi d'un niveau supérieur avant 1990 parce que les données ne les distinguaient pas des autres «entrées par ruse au domicile». Même chose pour les vols de voitures qui n'étaient pas distingués des vols de deux roues motorisés. On voit également que le trafic de drogues n'était pas distingué de l'usage, ni même de l'usage/revente introduit par des décrets plusieurs années après la loi de 1970 sur la pénalisation de l'usage de stupéfiants. D'autres catégories d'infractions n'étaient pas distinguées et ne sont donc pas chiffrées avant 1990. 
environ le double de celle des étrangers résidant sur le territoire métropolitain (5,6\%). Toutefois, comme le rappellent Robert et al. (1994 : 67), cette comparaison n'a pas véritablement de sens dans la mesure où, premièrement, les données policières concernent la totalité des étrangers mis en cause, qu'ils soient résidents en France ou bien touristes, saisonniers, frontaliers et clandestins; deuxièmement, la population étrangère résidant en France n'a pas la même structure démographique (par sexe et par âge) ni la même structure sociale (notamment par catégories socioprofessionnelles) que la population des nationaux. Les responsables des services statistiques du ministère de l'Intérieur reconnaissent du reste une source d'erreur dans leur recueil de chiffres annuel. Leur commentaire est le suivant :

les chiffres relatifs à la population étrangère recensée en France [...] sont établis sur la base des cartes de séjour en cours de validité, ils ne prennent en compte que les seuls étrangers ayant la qualité de résidents en France. Les étrangers circulant en France sous le seul couvert de leurs documents de voyage sont donc exclus de cette comptabilité. Cependant, au niveau des faits constatés, leur qualité d'étranger est prise en compte, ce qui introduit une marge d'erreur pour le calcul du taux de criminalité. Il n'existe actuellement aucune possibilité de calculer cette marge (Ministère de l'Intérieur, 1996 : 110).

C'est là une difficulté majeure que nous retrouverons plus loin dans des commentaires plus détaillés, mais sur laquelle il importait d'insister d'emblée, en répétant qu'elle est insurmontable en l'état actuel des données disponibles.

Si nous revenons à la proportion globale d'étrangers mis en cause par les services de police et de gendarmerie, il faut considérer à présent que ce pourcentage global cache de fortes disparités. Les étrangers sont à peine surreprésentés parmi les auteurs de vols de voiture, d'incendies, de destructions et de dégradations de biens publics ou privés, d'infractions contre la famille et l'enfant, et ils ne sont pas beaucoup plus souvent accusés de cambriolages, de vols dans les voitures (vols à la roulotte), d'escroqueries, de faux et de contrefaçons et d'agressions sexuelles autres que les viols. L'écart se creuse par contre avec des infractions comme les violences et outrages envers les

de $12,3 \%$ en 1999. Cela étant, on peut observer (voir tableau 3) que l'évolution dans le temps semble indiquer une réduction tendancielle de l'implication d'étrangers dans les catégories d'infractions les plus graves : viols, homicides, trafic de drogue, port et détention d'armes prohibées. Une seule exception à cette tendance : le proxénétisme, dont la part des étrangers dans les mis en cause connaît une augmentation très forte dans la deuxième moitié des années 1990. Mais il faut sans doute relativiser ces mouvements de court terme qui sont liés aux évolutions des filières d'approvisionnement du proxénétisme et de leur répression (on parle ainsi beaucoup depuis quelques années des nouvelles filières venues des pays d'Europe de l'Est). 
policiers, les ports et détentions d'armes prohibées, les viols, les vols avec violence mais sans arme à feu ou encore les homicides. Il atteint son maximum avec les trafics de drogues, les petits vols et le proxénétisme. Pour rechercher les faits les plus marquants dans cette liste d'infractions, il faut s'intéresser à la fois aux catégories d'infractions dans lesquelles la surreprésentation est la plus importante (quatrième colonne du tableau 2) et aux catégories dans lesquelles le nombre de personnes impliquées est le plus élevé (deuxième et troisième colonnes du tableau 2). Faisons prévaloir le nombre, sans oublier l'autre question. Il nous semble alors que six constats principaux se dégagent (ce sont les lignes en italiques dans le tableau 2). En ordre décroissant d'importance, notons donc que :

1) environ un étranger mis en cause sur buit est poursuivi pour vol à l'étalage;

2) environ un étranger mis en cause sur sept est poursuivi pour coups et blessures non mortels (autrement dit pour bagarres);

3) un peu plus d'un quart du total des personnes poursuivies pour trafics de drogues sont des étrangers (on verra les problèmes spécifiques qui se posent ici);

4) près de la moitié des mis en cause pour vol à la tire sont étrangers;

5) les étrangers sont nettement surreprésentés en matière d'bomicide et de tentative d'homicide, même s'il s'agit de petits nombres d'affaires;

6) les étrangers sont nettement surreprésentés en matière de proxénétisme, mais il s'agit ici d'affaires très peu nombreuses.

Les logiques sélectives du travail de police interdisent-elles toute interprétation sociologique de ces données? La question est délicate. À l'évidence, ces logiques sélectives expliquent directement la surreprésentation des étrangers dans la catégorie policière des «violences et outrages à dépositaires de l'autorité » (c'est-à-dire à l'endroit des policiers). C'est là, en effet, la conséquence la plus directe des pratiques policières de contrôle au faciès. Ces constats relativisent aussi en partie la part des étrangers dans certains petits vols élucidés en flagrant délit (c'est le cas en particulier du vol à l'étalage, dont on a déjà parlé). Les recherches mentionnées à propos de la consommation et du trafic de drogues invitent à la prudence sur cet autre sujet, d'autant que la statistique de police ne précise pas les pays d'origine des auteurs poursuivis, ni le fait que ces derniers résident ou non en France, de façon licite ou illicite. En réalité, nombre de personnes poursuivies pour trafic sont interpellées par les services de la 
douane aux frontières et dans les aéroports et, bien souvent, ne sont pas résidentes en France. Soulignons par ailleurs la confusion dans la même catégorie statistique entre trafic international et revente locale, deux phénomènes qui sont parfois liés dans une même filière, mais qui sont souvent nettement distincts ${ }^{10}$. Des recherches fondées sur le dépouillement d'affaires jugées à Bobigny, à Nanterre et à Lille indiquent nettement que, au sein des étrangers arrêtés par la police pour trafic de drogues, on rencontre d'une part des clandestins issus majoritairement de pays africains, qui vivent dans la précarité au bas de l'échelle du trafic, d'autre part des ressortissants de pays exportateurs de cannabis (notamment le Maroc) qui travaillent souvent dans le cadre de réseaux mixtes dans lesquels les positions dominantes sont souvent tenues par des Français d'origine française (Duprez et Kokoreff, 2000; Duprez et al., 2001).

\section{Les étrangers résidant en France:} une délinquance de pauvres?

Cela étant, certaines des données policières sont trop importantes pour ne pas correspondre à quelques réalités de la délinquance elle-même. L'essentiel réside alors dans l'interprétation qui en est donnée. Celle-ci nous semble assez aisée à suggérer. Du point de vue du nombre (la masse des quelques 90000 mises en cause), la surdélinquance des étrangers résidant en France semble être essentiellement une surdélinquance de miséreux : petits voleurs à la tire et à l'étalage, petits revendeurs de drogues, bagarreurs et parfois meurtriers. Ceci est confirmé aussi par une recherche récente portant sur une population d'une bonne centaine de meurtriers jugés en cours d'assises dans les années 1990 dans un département de la banlieue parisienne (Mucchielli, 2003). S'agissant d'un crime où l'effet du «tri ethnique» est certainement particulièrement faible, voire inexistant, il ressort sans ambiguiité que les étrangers sont surreprésentés parmi ces criminels. Leur part dans cet échantillon de criminels correspond au double de leur poids démographique dans la région étudiée. Parmi les auteurs étrangers concernés, ceux originaires du Maghreb et du Portugal sont les plus nombreux, ce qui correspond aussi à leur importance démographique dans le département. Enfin, il apparaît

10. Du coup, la distinction entre les catégories policières de «trafic» et d' «usage/revente» est incertaine. Or, les écarts entre ces deux catégories concernant la part des étrangers parmi les mis en cause sont très importants $(27,1 \%$ pour le trafic, $7,9 \%$ pour l'usage/revente en 2000). 
également avec force que ces criminels étrangers appartiennent presque exclusivement aux milieux populaires précarisés, comme en témoigne leur activité professionnelle (plus de la moitié sont des inactifs ou des chômeurs, les autres sont essentiellement des ouvriers), le type (précaire voire très précaire) et le lieu (souvent les grands ensembles les plus dégradés de la région) de leur logement.

Pour le reste, la délinquance des étrangers relève sans doute essentiellement de ce que les policiers appellent la «grande criminalité» (trafics divers, proxénétisme, escroqueries à grande échelle) et qui est sans doute liée à des organisations délinquantes dont les chefs ne résident pas nécessairement en France. Faute de renseignements, nous n'en traiterons pas davantage.

Notre interprétation principale (non exclusive, donc) est ainsi que la surreprésentation des étrangers résidant en France dans les personnes mises en cause par la police et la gendarmerie est fortement liée à leurs conditions de vie. En France, les ressortissants des pays africains - et leurs enfants, qu'ils aient ou non acquis la nationalité française - sont globalement dans une situation socioéconomique précaire, voire très précaire. Ils appartiennent d'abord massivement aux milieux populaires. Au recensement de 1990, les actifs étrangers originaires des pays du Maghreb sont ouvriers dans $66,5 \%$ des cas, employés dans $15,8 \%$ et chômeurs n'ayant jamais travaillé dans $4,2 \%$ des cas (INSEE, 1994). Le cumul de ces trois catégories donne $86,5 \%$, à quoi s'ajoutent $5,2 \%$ d'artisanscommerçants (restaurateurs, épiciers, etc.) rarement fortunés. Au total, on peut donc estimer qu'ils appartiennent aux milieux populaires dans environ $90 \%$ des cas (contre 60 à $65 \%$ dans la population française). Les étrangers sont même surreprésentés dans les métiers ouvriers les moins qualifiés et les plus pénibles ${ }^{11}$. Ils sont ensuite nettement surreprésentés parmi les travailleurs en intérim ${ }^{12}$ et en contrat à durée déterminée ${ }^{13}$. Ils sont enfin beaucoup plus touchés par le chômage. Dans l'enquête «Emploi» de l'INSEE en 1992, le taux de chômage des Français était de $9,5 \%$, celui des étrangers, de $18,6 \%$ mais il montait à $29,6 \%$ chez les

11. Si la part des étrangers dans la population active en 1991 était de $6,8 \%$, ils constituaient presque le double $(13,1 \%)$ des travailleurs victimes d'accidents du travail et presque le triple des travailleurs accidentés avec incapacité permanente (17,6\%) (INSEE, 1994).

12. L' «intérim» est un travail qui correspond généralement à un remplacement, pour une durée limitée (quelques semaines ou quelques mois), à temps complet ou partiel.

13. Un «contrat à durée déterminée», comme son nom l'indique, est une embauche pour une durée limitée, qui n'offre aucune promesse pour la suite et ne donne droit à aucune indemnité lorsqu'il prend fin. 
Maghrébins et, parmi ces derniers, le taux de chômage de la tranche des 15-24 ans atteignait 50,6\% (INSEE, 1994). La même enquête, datée de mars 2000, indique que ces ordres de grandeur n'ont pas changé. Le taux de chômage des étrangers $(20 \%)$ est le double du taux global des actifs (10\%), le triple (30\%) si l'on exclut les ressortissants de pays de l'Union européenne. Or, une recherche quantitative récente a pu montrer l'existence d'une corrélation très significative entre les vols et le taux de chômage des jeunes de moins de 25 ans non diplômés (Lagrange, 2001a).

L'enquête sur les revenus fiscaux de l'INSEE (1997) indique de son côté que, si $7 \%$ de l'ensemble des ménages résidant en France vivent sous le seuil de pauvreté, cette proportion s'élève à $25 \%$ pour les ménages dont le chef possède la nationalité algérienne, marocaine ou tunisienne (Hourriez et al., 2001). Cette situation, de plus, ne cesse de s'aggraver. Dans la région parisienne (île-de-France), où résident environ $12 \%$ de l'ensemble des étrangers, ces derniers représentaient $18 \%$ des ménages les plus pauvres en 1978. En 1996, cette proportion s'élève à $32 \%$ (Observatoire national de la pauvreté et de l'exclusion sociale, $2002: 80-81$ ).

Logiquement, cette population pauvre se concentre dans les quartiers qui font l'objet des politiques de la ville (Castellan et al., 1992). En 1992, un peu plus de 500 quartiers faisaient l'objet d'un contrat de ville et regroupaient environ 3 millions d'habitants. Leurs principales caractéristiques démographiques étaient la surreprésentation des étrangers (18\%, soit trois fois plus que sur l'ensemble du territoire métropolitain), des jeunes de moins de 20 ans $(33 \%$ contre $26 \%$ dans tout le territoire métropolitain) et des familles nombreuses (de 6 personnes ou plus dans le ménage (7,5\% contre 3,2\% dans tout le territoire métropolitain)). Plus précisément encore, dans ces quartiers la part d'étrangers parmi les moins de 15 ans atteignaient $21,6 \%$ (contre $7 \%$ dans tout le territoire métropolitain). Les «zones urbaines sensibles», créées par le Pacte de relance de la ville (1996), regroupent aujourd'hui près de 4,5 millions de personnes, avec des caractéristiques et des proportions globalement similaires, à l'exception notable d'un taux de chômage des jeunes qui a nettement progressé dans la décennie 1990 (Le Toqueux et Moreau, 2002).

En définitive, la surdélinquance des étrangers résidant en France ressemble à une version moderne de la surdélinquance traditionnelle des milieux sociaux les plus précarisés dans une société industrielle, telle qu'on peut en trouver des échos dans la littérature savante et la presse depuis le $\mathrm{XIX}^{\mathrm{e}}$ siècle. La liste des problèmes liés à ces conditions de vie précarisées est classique : familles fréquemment déstabilisées par le chômage et les acci- 
dents du travail chez les pères, précarité des conditions de vie générales qui bloque l'accès à de nombreuses ressources et génère un fort sentiment de frustration chez les enfants, familles nombreuses vivant dans peu d'espace, d'où un investissement de la rue comme terrain de jeux et d'expériences pour les enfants (de là découlent à la fois une intense sociabilité juvénile et des problèmes de surveillance parentale), faible niveau scolaire ne permettant pas un soutien à la scolarité des enfants ${ }^{14}$, fréquence plus importante des cas de troubles psychologiques et d'alcoolisme chez les parents. Cependant, à ces problèmes sociaux classiques s'ajoutent, pour certaines catégories d'étrangers, ceux liés à des stigmates spécifiques. Pour tenter de le comprendre, il faut analyser à présent la situation des jeunes hommes issus de l'immigration africaine, nés en France de parents étrangers.

\section{Une surdélinquance des «jeunes issus de l'immigration »?}

Dans le débat public français, c'est aujourd'hui moins les étrangers que les jeunes Français nés de parents ou de grands-parents étrangers, et tout particulièrement les jeunes d'origine maghrébine, voire plus largement africaine, qui sont l'objet d'une très fréquente suspicion, ancrée notamment dans une présomption de dangerosité. Le système statistique français actuel ne permet pas de connaitre la situation des Français nés de parents étrangers avec la même précision que la situation des étrangers ${ }^{15}$.

14. En 1994, 2,3 millions d'adultes vivant en France métropolitaine présentent des difficultés à parler, lire, écrire ou maîtriser le français dans la vie courante; ces personnes représentent 3,2\% de l'ensemble des adultes dont le français est la langue maternelle mais 30,1\% de ceux dont il ne l'est pas (Bodier et Chambaz, 1995).

15. La prise en compte de la variable «origine» est depuis quelques années l'objet de débats parfois houleux au sein de la communauté scientifique, notamment chez les démographes. Indiquons que nous sommes pour notre part de ceux qui pensent que ces polémiques sont inutiles, que l'origine nationale est une donnée comme une autre qui ne contredit nullement le principe de la citoyenneté une et indivisible (pas plus que l'interrogation sur l'origine régionale ou locale par exemple), et que la censure morale de la variable «origine» n'est pas fondée, car aucune statistique n'a jamais convaincu personne d'être raciste ou antiraciste (Mucchielli, 1999a : 115-117). Par ailleurs, nous partageons l'argument selon lequel ces statistiques, loin d'entériner ou de nourrir un quelconque racisme, seraient au contraire des éléments utiles pour analyser les discriminations dont les personnes d'origine étrangère sont fréquemment victimes dans la vie sociale (Simon, 1999: 111 et 114). Précisons enfin que, en France, ces débats statistiques n'ont qu'une portée limitée pour les sciences sociales, dans la mesure où l'ethnicité est largement une construction sociale locale et non une donnée invariable généralisable à l'ensemble du territoire (voir par exemple un point du débat et une illustration empirique : Rinaudo, 1999), ce que le présent texte s'efforce de montrer aussi à sa manière. 
Pour s'enquérir de la délinquance des "jeunes issus de l'immigration», il faut donc rechercher des données d'enquêtes construites par les chercheurs, qu'il s'agisse de données qualitatives ou quantitatives.

\section{Données d'enquêtes}

Dans un sondage de délinquance autorévélée réalisé en 1999 auprès d'un échantillon de 2288 jeunes de 13 à 19 ans, Sebastian Roché (2001) constate une nette surdélinquance des jeunes dont les deux parents sont étrangers et, en leur sein, une légère surreprésentation des jeunes maghrébins par rapport aux autres étrangers. Il note que «les jeunes d'origine maghrébine cumulent un certain nombre de facteurs associés à la délinquance : une plus faible supervision parentale, une résidence plus fréquente dans le parc HLM hors centre-ville, un niveau de revenu et de scolarisation faible des parents, un absentéisme scolaire plus élevé» (p. 221). Il insiste ensuite sur le problème des mauvaises relations des jeunes d'origine maghrébine avec la police, sans toutefois donner les éléments nécessaires à la mesure de l'incidence de ce problème sur la commission d'actes délinquants. Mais la limite principale de cette étude réside dans son caractère purement quantitatif et global, qui écrase les effets de contextes locaux.

Les recherches de Hugues Lagrange (2001b), réalisées à la fin des années 1990, sont plus intéressantes dans cette perspective. Ce chercheur a procédé en analysant systématiquement les patronymes d'échantillons de délinquants identifiés par la police, d'une part dans une ville moyenne de province comme Amiens, d'autre part dans le pays Mantois (Mantes-LaJolie, Mantes-La-Ville et Les Mureaux), zone dite «très sensible» de l'agglomération parisienne concentrant typiquement les handicaps économiques et sociaux, les grands ensembles et leur population massivement étrangère et d'origine étrangère (ce que Dubet et Lapeyronnie (1992) appellent les «quartiers d'exil»). Les résultats sont nets. À Amiens, il n'y a pas de surdélinquance des jeunes issus de l'immigration africaine. Ces derniers ont un comportement analogue à celui des autres jeunes issus de familles pauvres (une partie d'entre eux est, pour l'essentiel, bagarreuse et voleuse). Notre interprétation est donc confirmée. Mais dans le Mantois, la situation est différente. Il y existe une forte surdélinquance des jeunes issus de l'immigration africaine (autant d'Afrique noire que du Maghreb) ${ }^{16}$. Lagrange conclut que les comportements délinquants ou rebelles des jeunes

16. De même que, dans une moindre mesure, des jeunes issus de l'immigration portugaise. 
issus de l'immigration varient donc fortement selon le contexte local. Il ne se demande pas toutefois si la discrimination policière ne varie pas elle aussi selon les contextes locaux. Or, l'on peut assez aisément faire l'hypothèse générale que les quartiers pauvres, qui concentrent le plus de population étrangère et d'origine étrangère et qui connaissent par ailleurs une consommation et des systèmes de revente et/ou de trafic de cannabis particulièrement développés, connaissent davantage de tensions entre jeunes et policiers. Les recherches sur les violences policières soulignent en effet la forte surreprésentation des jeunes hommes étrangers et d'origine étrangère parmi les victimes de ces violences, en particulier dans les affaires liées à la consommation et/ou à la revente de drogues (Jobard, 2002 : 210-211). Par ailleurs, l'attitude locale des forces de police dépend certainement aussi de leur relation avec les autres acteurs institutionnels locaux, de leur relation avec les élus locaux (qui peuvent exercer une pression en des sens opposés) et avec la préfecture, et enfin, de la personnalité et de l'expérience des cadres policiers locaux.

Quoi qu'il en soit, la question se déplace donc vers la recherche de ce qui caractérise ces contextes et touche de façon spécifique (ou du moins principale) les jeunes issus de l'immigration. À ce moment de son analyse, $\mathrm{H}$. Lagrange évoque alors des «violences collectives» et leur dimension d' «affirmation identitaire», signalant par ailleurs la prégnance de l'Islam dans ces quartiers, en tant qu'il offre à ces jeunes la possibilité de retrouver une dignité. L'explication mérite toutefois d'être davantage précisée, à la fois dans ses mécanismes et au regard de la nature des comportements délinquants.

\section{Éléments psychosociologiques sur la délinquance des jeunes issus de l'immigration africaine}

Les explications culturalistes constituent un réductionnisme avec lequel les sciences sociales ont mis longtemps à prendre leurs distances, en France comme aux États-Unis. Nous avons vu ce qu'il en était de la fortune du thème du déracinement. Une autre explication courante s'agissant des «jeunes issus de l'immigration» consiste à mettre en avant leur «double culture» présentée comme constituant fatalement une source de contradictions et de conflits, donc de perturbations psychologiques et de déviances. Dans sa généralité, cette idée est fausse, d'une part parce qu'elle repose sur un modèle opposé de parfaite homogénéité culturelle qui n'a pas de sens dans un très vieux pays d'immigration comme la France, d'autre part parce 
qu'elle réduit la construction de la personnalité sociale à la problématique culturelle, passant ainsi sous silence celle de l'intégration socioéconomique. Ce qui est exact, c'est que la question des origines et le rapport à la culture des parents se pose fatalement à la «deuxième génération» d'immigrés. Mais de quelle manière? Les travaux historiques comme les enquêtes sociologiques récentes soulignent que, en France, les jeunes Français nés de parents étrangers (qu'il s'agisse des vagues d'immigration italiennes et espagnoles de la première moitié du $\mathrm{XX}^{\mathrm{e}}$ siècle, ou aussi bien des vagues d'immigration maghrébines de l'après-guerre) ont globalement toujours connu en même temps un double processus : d'une part une très forte pression de conformité à l'égard du pays d'accueil, amenant à rejeter précocement la culture d'origine de leurs parents, perçue comme inférieure ou archaïque, au point de pouvoir en ressentir de la bonte, d'autre part un mouvement de contestation visant à rejeter le stigmate et à faire accepter dans l'espace public leur spécificité. S'agit-il d'une contradiction? Dans son remarquable bilan, Noiriel (1988: 211-245) laissait la question «ouverte». Quant à nous, il nous semble qu'il n'y a pas de contradiction entre ces deux attitudes qui procèdent de la même source : le fait que la culture du pays d'origine soit stigmatisée dans le pays d'accueil. Toutes les contradictions prétendues insurmontables liées à la «double culture» se ramènent donc selon nous à un unique problème central : la gestion du stigmate, la dévalorisation identitaire qu'il provoque dès l'enfance (Vinsonneau, $1996^{17}$; Esterle-Hedibel, 1999) et, inséparablement, les réactions de défense et les stratégies de revalorisation de soi qu'il induit plus ou moins (Malewska, 1982; 1991; Camilleri et al., 1990). Et «plus ou moins», en fonction de quoi? Il nous semble que cette gestion du stigmate est liée principalement à trois éléments : primo la réussite ou non de l'intégration socioéconomique, intégration dont la phase cruciale est le parcours scolaire du jeune, secundo les déterminants familiaux que sont l'histoire familiale (incluant la nature du projet migratoire et le discours tenu sur lui), la réussite professionnelle des parents, l'attitude de ces derniers vis-à-vis de l'école et plus largement des valeurs du pays d'accueil, tertio les éléments du contexte immédiat de vie, en particulier les effets plus ou moins stigmatisants de l'habitat, les expériences plus ou moins précoces et intenses du racisme et les influences exercées par les groupes de pairs.

17. Cette recherche de psychologie sociale conclut que le repérage des stigmatisations ethniques (ou construites comme telles) commence à partir de l'âge de 4 ans et se structure une première fois entre l'âge de 5 et 7 ans. 


\section{Les contextes favorables au développement} d'une surdélinquance des jeunes issus de l'immigration

Dès lors que l'analyse sociologique a pu rompre avec les réductions culturalistes et mettre en évidence l'élément central qui distingue la construction identitaire des jeunes issus de l'immigration africaine des autres jeunes Français, la question se déplace vers la recherche des contextes qui peuvent engendrer une surdélinquance des jeunes issus de l'immigration.

Dans la société française actuelle, ce contexte est celui des grands ensembles dégradés des banlieues des grandes agglomérations (et parfois des quartiers périphériques des grandes villes), où la population étrangère et d'origine étrangère est souvent majoritaire, où le taux de chômage est particulièrement élevé, où la proximité avec des centres-villes attractifs accentue encore la frustration, où la stigmatisation des lieux (leur mauvaise réputation) accentue encore celle des populations qui les habitent et contribue fortement aux discriminations dans le rapport aux institutions et au marché de l'emploi (Duprez, 1997). Nous ferons aussi l'hypothèse que l'élément déterminant est moins ce contexte en lui-même, photographié à un moment donné, que le sentiment couramment partagé (chez les personnes qui habitent ces quartiers) qu'il constitue une fatalité, voire un piège duquel l'on a peu de chances de s'extraire. À partir de la fin des années 1980, pour des raisons économiques et peut-être surtout politiques (l'échec des mobilisations collectives des années 1982-1986), le facteur temps produit un effet inverse par rapport au modèle de trajectoire couramment partagé dans le monde ouvrier et chez les immigrés de la première génération (Mucchielli, $2002: 107$ et suivantes) ${ }^{18}$. Comme le dit Khosrokhavar (1997 : 186), «pour la première fois, une génération ne croit plus à l'utopie de l'ascension sociale étalée dans le temps». Cumulée avec la stigmatisation dans l'espace public et dans le rapport aux institutions, cette perspective d'un destin social misérable produit d'intenses sentiments de frustration, d'exclusion et de mépris subi collectivement. C'est dans ce sens que nous proposons de parler de sentiments de victimation collective pour analyser la mentalité de ces jeunes qui disent souvent eux-

18. On perçoit par exemple ce processus générationnel et la force de ce modèle de trajectoire à travers ce témoignage d'un membre de la Commission nationale de prévention de la délinquance, qui disait encore en 1987 : «Il y a 15 ans, j'étais responsable d'un foyer d'éducation surveillée à Nogent. La moitié de mon temps était prise par de jeunes Espagnols ou Portugais. Je n'en ai plus vu quand leurs pères sont devenus chefs de chantiers. Peut-être faudra-t-il quelques années pour ne plus s'occuper de jeunes Maghrébins» (cité par Taïeb, $1998: 353)$. 
mêmes vivre dans des ghettos, et pour souligner que ces représentations collectives se rigidifient au point de confiner parfois à une théorie du complot : le complot d'une société injuste et raciste (Mucchielli, 1999b).

Les comportements délinquants juvéniles trouvent certainement dans ces représentations de puissants arguments déculpabilisateurs ${ }^{19}$. Mais de quels comportements délinquants s'agit-il précisément? Le sentiment de vivre dans un ghetto ne semble pas encourager en soi la violence contre des personnes privées comme le meurtre ou le viol. L'enfermement dans l'espace micro local peut générer par contre davantage de violences entre jeunes de villes ou de quartiers frontaliers. De fait, la forte hausse des coups et blessures non mortels dans les statistiques de police depuis la fin des années 1980, corroborée sur ce point par les enquêtes de victimation (Robert et al., 1999), traduit sans doute cette intensification des affrontements juvéniles, souvent en petits groupes. Ensuite, on peut remarquer que les plus fortes hausses de la délinquance enregistrée des mineurs, au cours des années 1990, concernent avant tout les vols (notamment les vols de voiture dont on sait qu'ils peuvent avoir diverses significations, dont celle de se venger d'un groupe adverse (Esterle-Hedibel, 1996)), les bagarres (qui nous renverraient à nouveau à ces processus de conflits locaux), les consommations et trafics de drogues, les destructions et dégradations de biens publics et les «outrages et violences» envers des agents de la force publique (Aubusson de Cavarlay, 1997). Et, encore une fois, si ces données institutionnelles ne constituent en aucune façon une mesure exacte du niveau et de l'évolution des comportements, elles entérinent néanmoins certaines réalités sociales dont les institutions se préoccupent plus particulièrement. Ceci amène à souligner deux autres dimensions probables de cette surdélinquance localisée des jeunes issus de l'immigration. Une première est économique. Le sentiment que toute perspective d'insertion économique et sociale est bouchée constitue logiquement un puissant facteur facilitant l'investissement de l'économie souterraine dans ses composantes classiques (par exemple les vols, recels et reventes de pièces détachées de voitures) ou plus récentes (le développement du trafic et de la revente de cannabis depuis les années 1980). Une seconde est sa dimension anti-institutionnelle. Nous pouvons parler ici d'une «violence contre les institutions» qui regroupe diverses formes de dégradation et de violence exercées à l'encontre des biens et des personnes qui

19. Nous nous inspirons en partie ici des analyses classiques de Sykes et Matza (1957) sur les «techniques de neutralisation» de la culpabilité par les délinquants. 
symbolisent les institutions. Ceci concerne de nombreux acteurs (policiers, transporteurs collectifs, écoles et bâtiments publics, parfois pompiers), mais l'un d'eux joue un rôle décisif en raison de la fréquence et de la nature de ses rapports avec les jeunes des quartiers concernés : c'est la police. «Les tensions avec la police sont une dimension majeure de l'expérience urbaine des jeunes des quartiers pauvres», constate Michel Kokoreff (2003 : 144). De fait, nombre de recherches indiquent clairement que les relations entre jeunes d'origine africaine et police dans ces quartiers dits très sensibles fonctionnent avec des cycles de provocations, ripostes, représailles, etc., entretenus de part et d'autre (pour une synthèse récente : Esterle-Hedibel, 2002). C'est bien dans ces contextes qu'éclatent parfois des «émeutes» qui cristallisent les représentations collectives en question et cette «revendication essentielle de dignité et de justice» (Lapeyronnie, $1993: 263)^{20}$. Comme l'expliquent également Bachmann et Le Guennec (1996 : 355), au delà des incidents qui précipitent le déclenchement d'une émeute, «contre qui se battent les émeutiers? Contre un ennemi sans visage. Contre ceux qui les nient quotidiennement, les condamnent à l'inexistence sociale et leur réservent un avenir en forme d'impasse. L'environnement quotidien est tissé de méfiance et d'hostilité; le futur est bouché. Aucun allié. Aucune issue. [...] Ces deux sentiments forts, la sensation de l'impasse et la conscience du mépris, sont toujours à la racine des fureurs banlieusardes».

\section{Conclusions}

Il n'est pas exagéré de dire que, dans l'univers ordinaire des représentations sociales ${ }^{21}$, les jeunes d'origine africaine («Blacks» et «Beurs») constituent une figure type du jeune délinquant, tandis que les quartiers d'habitat social dans lesquels ils sont concentrés font figure de zones dangereuses (Rey, 1996; Boucher, 2001). Sans doute, ces stéréotypes

20. Ce qui n'exclut pas, comme dans le cas des débordements qui suivent parfois les manifestations politiques (Ricordeau, 2001), que les émeutes soient aussi des moments de défoulement et de transgressions que des petits groupes peuvent prévoir et organiser à leur profit.

21. Nous partageons cette définition de Michaud et Marc (1981:127) : «On peut définir une représentation sociale comme une image de certains éléments constitutifs de la réalité sociale, élaborée et partagée par une collectivité et qui contribue à orienter les conduites, les communications et les rapports sociaux ». Il nous semble que l'on peut relier ici plusieurs traditions théoriques tant cette définition des représentations sociales (qui puise sa source française dans la tradition durkheimienne) rejoint aisément les théories de Becker et de Goffman sur les processus d'étiquetage et de stigmatisation. 
ont-ils toujours visé l'étranger. À la fin du XIX ${ }^{\mathrm{e}}$ siècle, par exemple, les Italiens et les Belges firent l'objet d'une intense xénophobie et de nombreuses violences collectives (notamment au sein du monde ouvrier). En réalité, l'historien Gérard Noiriel (1988: 249 et suivantes) souligne que les trois crises économiques modernes qu'a connues la France (à la fin du XIX ${ }^{\mathrm{e}}$ siècle, dans les années 1930 et à partir du milieu des années 1970) ont suscité autant de vagues de xénophobie. Par ailleurs, ces problèmes se rencontrent aussi chez certains de nos voisins européens ${ }^{22}$. Dès lors, nous ne saurions dire si ces stéréotypes sont plus puissants s'agissant de la dernière grande vague d'immigration initiée par les entreprises françaises dans les années 1950. Ils présentent toutefois une certaine spécificité dans la mesure où ils prennent aussi leur source dans le passé colonial de la France, la relation de domination (militaire, politique et économique) qu'elle a imposée à d'autres civilisations et les sentiments de supériorité individuelle et collective qui ont toujours accompagné cette histoire. En ce sens, ces stéréotypes particulièrement dévalorisants sont une source de difficultés supplémentaires pour une population issue d'une vague d'immigration ouvrière déqualifiée et qui s'est retrouvée "piégée» par la crise économique au moment même où elle se stabilisait en France, par l'entremise notamment du regroupement familial. En orientant tant les pratiques des institutions que les représentations que des acteurs ont d'eux-mêmes, ces stéréotypes ressemblent alors à ces prophéties autoréalisatrices dont parlait si justement Robert Merton (1965 : 140-161).

Toutefois, même si tout jeune ayant ce profil doit se construire psychiquement en apprenant à gérer ce stigmate, cette construction ne le mène pas pour autant fatalement vers des pratiques et a fortiori une carrière délinquantes ${ }^{23}$. Ces pratiques et ces éventuelles carrières ne s'observent de façon spécifique (par rapport aux autres catégories de la population) que dans certains contextes locaux où les processus de ségrégation et de discrimination se cumulent et s'enracinent dans la durée, se transmettant entre générations. Pour le reste, les éléments déterminants de la délinquance juvénile des étrangers résidant en France et des Français

22. Nous pensons notamment à l'Allemagne et à sa forte immigration turque, qui suscite des débats similaires (voir par exemple Güller (1999), qui procède également à une analyse des données policières et démographiques très proche de la nôtre).

23. Rappelons qu'au recensement de 1990, un jeune de moins de 17 ans sur sept vivait dans un ménage dont le chef était immigré (Tribalat, 1993). Ce dernier était dans environ $40 \%$ des cas originaire d'un pays du Maghreb, puis, en ordre décroissant d'importance, portugais, africain noir, italien, espagnol, turc et asiatique. 
nés de parents étrangers demeurent des problèmes familiaux et scolaires qui ne sont pas propres à ces populations, mais qui semblent au contraire comparables aux problèmes posés jadis par des populations françaises issues de l'exode rural ou par d'autres populations ouvrières étrangères en période de crise économique. Il nous semble du reste que les mécanismes sociaux généraux proposés dans cet article se retrouvent également en bonne partie dans d'autres pays occidentaux ${ }^{24}$.

\section{Références}

Aubusson de Cavarlay, B. (1997). La place des mineurs dans la délinquance enregistrée. Les cabiers de la sécurité intérieure, 29, 17-38.

Bachmann, C., \& Le Guennec, N. (1996). Violences urbaines. Ascension et chute des classes moyennes à travers cinquante ans de politique de la ville. Paris : Albin Michel.

Barré, M.-D., \& Godefroy, T. (1999). Le consommateur de produits illicites et l'enquête de police judiciaire. CESDIP-OFDT, étude ${ }^{\circ} 19$.

Bauer, A., \& Raufer, X. (1998). Violences et insécurité urbaines. Paris : PUF.

Becker, H. (1985 [1963]). Outsiders. Études de sociologie de la déviance. Paris : Métaillé.

Bodier, M., \& Chambaz, C. (1995). La difficile maitrise de la langue française. INSEE Première, 385, 1-4.

Boucher, M. (2001). Les représentations pusillanimes des quartiers d'habitat social, de la présence immigrée et de l'Islam en France. In M. Boucher (ed.), De l'égalité formelle à l'égalité réelle. La question de l'etbnicité dans les sociétés contemporaines (217-240). Paris : L'Harmattan.

Bousquet, R. (1998). Insécurité, nouveaux risques. Les quartiers de tous les dangers. Paris : L'Harmattan.

Brion, F., \& Tulkens, F. (1998). Conflit de culture et délinquance. Interroger l'évidence. Déviance et société, 22 (3), 235-262.

Bui Trong, L. (1998). Les rapports des jeunes avec l'autorité policière. MigrantsFormation, 112, 47-54.

Camilleri, C., Kastersztein, J., Lipianski, E., Malewska-Peyre, H., TaboadaLeonetti, I., \& Vasquez, A. (1990). Stratégies identitaires. Paris : PUF.

Castellan, M., Marpsat, M., \& Goldberger, M.-F. (1992). Les quartiers prioritaires de la politique de la ville. INSEE Première, 234, 1-4.

Cesari, J. (1997). Faut-il avoir peur de l'Islam? Paris : Presses de Sciences Po.

Choquet, M., \& Ledoux, S. (1994). Adolescents. Enquête nationale. Paris : Éditions de l'Inserm.

Desdevises, M.-C. (1998), Migration et délinquance. Les aspects criminologiques du phénomène migratoire. Revue internationale de criminologie et de police technique et scientifique, 3, 268-281

24. Nos conclusions rejoignent ainsi largement celles de Sampson et Lauritsen (1997) sur les États-Unis. 
Dubet, F., \& Lapeyronnie, D. (1992). Les quartiers d'exil. Paris : Seuil.

Duprez, D. (1997). Entre discrimination et désaffiliation. L'expérience des jeunes issus de l'immigration maghrébine. Annales de la recherche urbaine, 76, 79-88.

Duprez, D., \& Kokoreff, M. (2000). Le monde des drogues. Usages et trafics dans les milieux populaires. Paris : Odile Jacob.

Duprez, D., Kokoreff, M., \& Weinberger, M. (2001). Carrières, territoires et filières pénales. Pour une sociologie comparée des trafics de drogues. Paris : Observatoire français des drogues et des toxicomanies.

Esterle-Hedibel, M. (1996). Virées, incendies et vols de voitures : motivations aux vols et aux dégradations de voitures. Déviance et société, 20 (4), 119-139.

Esterle-Hedibel, M. (1999). La marque de l'étranger. La construction d'identités délinquantes chez les jeunes d'origine maghrébine. In J.-M. Bessette (ed.), Crimes et cultures (137-146). Paris : L'Harmattan.

Esterle-Hedibel, M. (2002). Jeunes des cités, police et désordres urbains. In L. Mucchielli \& P. Robert (eds), Crime et sécurité : l'état des savoirs (376-385). Paris : La Découverte.

Gaspard, F., \& Khosrokhavar, F. (1995). Le foulard et la République. Paris : La Découverte.

Güller, N. (1999). La criminalité des Turcs en Allemagne. In J.-M. Bessette (ed.), Crimes et cultures (237-252). Paris : L'Harmattan.

Hourriez, J.-M., Legendre, N., \& Le Verre, R. (2001). La pauvreté monétaire. Paris : INSEE «Synthèses », $\mathrm{n}^{\circ} 47$.

INSEE (1994). Les étrangers en France. Paris : INSEE, «Contours et caractères ». Jobard, F. (2002). Les violences policières. In L. Mucchielli \& P. Robert (eds), Crime et sécurité : l'état des savoirs (206-214). Paris : La Découverte.

Khosrokhavar, F. (1997). L'Islam des jeunes. Paris : Flammarion.

Kokoreff, M. (2003). La force des quartiers. De la délinquance à l'engagement politique. Paris : Payot.

Lagrange, H. (2001a). Crime et conjoncture économique. Revue française de sociologie, $42(1), 57-79$

Lagrange, H. (2001b). De l'affrontement à l'esquive. Violences, délinquances et usages de drogues. Paris : Syros.

Lapeyronnie, D. (1993). L'individu et les minorités. La France et la Grande-Bretagne face à leurs immigrés. Paris : PUF.

Lebon, A. (2000). Immigration et présence étrangère en France en 1999. Premiers enseignements du recensement. Paris : La Documentation française.

Le Toqueux, J.-L., \& Moreau, J. (2002). Les zones urbaines sensibles. INSEE Première, 835, 1-4.

Lévy, R. (1987). Du suspect au coupable. Le travail de police judiciaire. Paris-Genève : Méridiens Klincsieck - Médecine et Hygiène.

Lévy, R., \& Zauberman, R. (1998). La police et les minorités visibles : les contradictions de l'idéal républicain. In Y. Cartuyvels, F. Digneffe, A. Pirès \& P. Robert (eds), Politique, police et justice au bord du futur (287-300). Paris : L'Harmattan. 
Malewska, H. (1982) Crise d'identité et déviance chez les jeunes immigrés. Paris : La Documentation française.

Malewska-Peyre, H. (1991). La socialisation en situation de changement culturel. In H. Malewska-Peyre \& P. Tap (eds), La socialisation de l'enfance à l'adolescence (195-218). Paris : PUF.

Mary, F.-L., \& Tournier, P. (1998). La répression pénale de la délinquance des étrangers en France. Information - Prison - Justice, 84, 1-4.

Merton, R.K. (1965 [1957]). Éléments de théorie et de méthode sociologique. Paris : Plon.

Michaud, G., \& Marc, E. (1981). Vers une science des civilisations? Bruxelles : Complexe.

Ministère de l'Intérieur (1976). La criminalité en France en 1975. Paris : Ministère de l'Intérieur.

Ministère de l'Intérieur (1996). Aspects de la criminalité et de la délinquance constatées en France en 1995. Paris : La Documentation française.

Missaoui, L., \& Tarrius A. (1999) Naissance d'une mafia catalane? Perpignan : Le Trabucaire.

Monjardet, D. (1996). Ce que fait la police. Sociologie de la force publique. Paris : La Découverte.

Mucchielli, L. (1999a). Il n'y a pas de statistique raciste, seulement des interprétations. Mouvements, 3, 115-117.

Mucchielli, L. (1999b). Le rap et l'image de la société française chez les «jeunes des cités». Questions pénales, 2, 1-4.

Mucchielli, L. (2002). Violences et insécurité. Fantasmes et réalités dans le débat français. Paris : La Découverte, $2^{\mathrm{e}}$ édition.

Mucchielli, L. (2003; à paraître). Les caractéristiques démographiques et sociales des meurtriers et de leurs victimes. Un test dans la France des années 1990.

Noiriel, G. (1988). Le creuset français. Histoire de l'immigration, XIX $X^{e}-X X^{e}$ siècle. Paris : Seuil.

Observatoire géopolitique des drogues (1996). Atlas mondial des drogues. Paris : PUF.

Observatoire national de la pauvreté et de l'exclusion sociale (2002). Rapport 2001-2002. Paris : La Documentation française.

Ocqueteau, F., \& Pottier, M.-L. (1995). Vigilance et sécurité dans les grandes surfaces. Paris : l'Harmattan.

Rey, H. (1996). La peur des banlieues. Paris : Presses de Sciences-Po.

Ricordeau, G. (2001). Pourquoi cassent-ils? Présentation des discours et motivations des casseurs. Déviance et société, 25 (2), 165-173.

Rinaudo, C. (1999). L'ethnicité dans la cité. Jeux et enjeux de la catégorisation ethnique. Paris : l'Harmattan.

Robert, P. (1995). L'uguaglianza degli imputati di fronte alla giustizia penale. In A. Cottino \& C. Sarzotti (eds), Diritto, uguaglianza e giustizia penale (181188). Torino : L'Harmattan Italia.

Robert, P. (1999). Le sociologue, la culture et le crime. In J.-M. Bessette (ed.), Crimes et cultures (29-59). Paris : L'Harmattan. 
Robert, P., Aubusson de Cavarlay, B., Pottier, M.-L., \& Tournier, P. (1994). Les comptes du crime. Les délinquance en France et leur mesure. Paris : l'Harmattan.

Robert, P., Zauberman, R., Pottier, M.-L., \& Lagrange, H. (1999). Mesurer le crime. Entre statistiques de police et enquêtes de victimation (1985-1995). Revue française de sociologie, 40 (2), 255-294.

Roché, S. (2001). La délinquance des jeunes. Les 13-19 ans racontent leurs délits. Paris : Seuil.

Sampson, R., \& Lauritsen, J. (1997). Racial and ethnic disparities in crime and criminal justice in the United States. In M. Tonry (ed.), Ethnicity, crime, and immigration. Comparative and cross-national perspectives (311-374). Crime and Justice. A Review of Research, 21 : University Press of Chicago.

Sayad, A. (1981). Santé et équilibre social chez les immigrés. Psychologie médicale, 11, 1747-1775.

Sayad, A. (1997). L'immigration et la «pensée d'État». Réflexions sur la double peine. In S. Palidda (ed.), La construction sociale de la déviance et de la criminalité parmi les immigrés en Europe (11-29). Luxembourg : Conseil de l'Europe, COST Migrations.

Sellin, T. (1928). The negro criminal. A statistical note. Annals of the American Academy of Political and Social Science, 229, 11-29.

Sellin, T. (1935). Race prejudice in the administration of justice. American Journal of Sociology, 41, 212-217.

Simon, P. (1999). Sciences sociales et racisme : où sont les docteurs Folamour? Mouvements, 3, 111-114

Sternhell, Z. (1985 [1972]). Maurice Barrès et le nationalisme français. Bruxelles : Complexe.

Sykes, G., \& Matza, D. (1957). Techniques of neutralization. A theory of delinquency. American Sociological Review, 2, 664-670.

Taïeb, E. (1998). Immigrés : l'effet générations. Paris : Éditions de l'Atelier.

Tournier, P. (1997). Nationality, crime and criminal justice in France. In M. Tonry (ed.), Ethnicity, crime, and immigration. Comparative and cross-national perspectives (523-551). Crime and Justice. A Review of Research, 21 : University Press of Chicago.

Tournier, P., \& Robert, P. (1990). Étrangers et délinquance : les chiffres du débat. Paris : l'Harmattan.

Tribalat, M. (1993). Les immigrés au recensement de 1990. Population, 6, 19111946.

Veitl, P. (1994). La géographie sociale du crime et la pratique réformatrice d'Henri Joly. In L. Mucchielli (ed.), Histoire de la criminologie française (269285). Paris : L'Harmattan.

Vinsonneau, G. (1996). L'identité des jeunes en situation inégalitaire. Le cas des maghrébins en France. Paris : L'Harmattan.

Zauberman, R. (1998). Gendarmerie et gens du voyage en région parisienne. Cabiers internationaux de sociologie, 4, 415-452. 